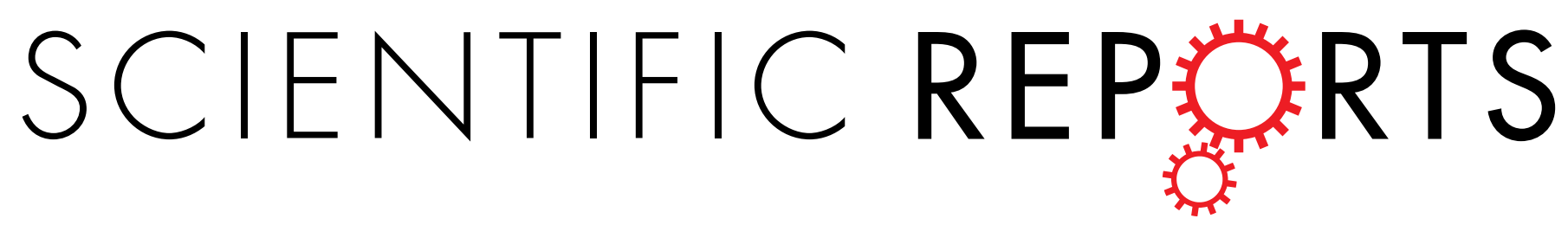

\title{
Corrigendum: A method for human teratogen detection by geometrically confined cell differentiation and migration
}

Jiangwa Xing, Yi-Chin Toh, Shuoyu Xu \& Hanry Yu

Scientific Reports 5:10038; doi: 10.1038/srep10038; published online 12 May 2015; updated on 03 August 2015

In the original version of this Article, there is a typographical error in Affiliation 1 which was incorrectly listed as Institute of Biotechnology and Nanotechnology, A ${ }^{\star S T A R, ~ T h e ~ N a n o s, ~ \# 04-01, ~} 31$ Biopolis Way, Singapore 138669, Singapore.' The correct affiliation is listed below:

Institute of Bioengineering and Nanotechnology, ${ }^{\star}{ }^{\star}$ TAR, The Nanos, \#04-01, 31 Biopolis Way, Singapore 138669, Singapore

This error has now been corrected in the PDF and HTML versions of the Article. 